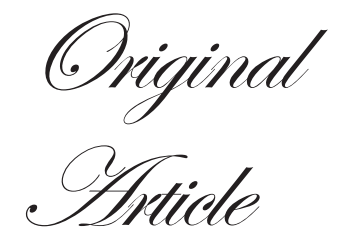

\title{
Risk of development of hepatocellular carcinoma in patients with NASH-related cirrhosis
}

\author{
DN Amarapurkar, M Dharod, Sonali Gautam, N Patel
}

\section{ABSTRACT}

Bombay Hospital and Medical

Background and aims: The risk of development of hepatocellular carcinoma (HCC) in hepatitis Research Centre, Mumbai, Maharashtra, India

\section{Correspondence:}

Dr Deepak Amarapurkar

Email: amarapurkar@gmail.com $\mathrm{B}$ virus (HBV) and hepatitis $\mathrm{C}$ virus (HCV) infection is well established and is being recognized increasingly in non-alcoholic steatohepatitis (NASH)-related cirrhosis. This study aimed to assess the risk of development of HCC in patients with NASH-related cirrhosis.

Methods: From January 2010 to October 2011, we prospectively enrolled 585 patients with liver cirrhosis (men:women ratio 4.4:1, mean age 50.1 \pm 6.1 years, aetiology HBV 19\%, HCV $14.2 \%$, NASH-related 7\%, cryptogenic cirrhosis $17.8 \%$, already diagnosed cirrhosis $48.2 \%$, and the remaining were newly diagnosed cases). The cumulative follow-up was for $5.9 \pm 0.5$, $6.1+0.8$ and $6.8+1.2$ years for $\mathrm{HBV}, \mathrm{HCV}$ and NASH-related cirrhosis, respectively. Patients with advanced cirrhosis, Child class $\mathrm{C}$ and associated comorbid conditions where survival was $<1$ year were excluded from the study. The remaining patients were followed up 6-monthly with ultrasound examination and alpha-fetoprotein (AFP) test. Patients suspected of HCC underwent triple-phase computed tomography (CT) scan and liver biopsy was done to confirm the diagnosis.

Results: A total of 54 patients developed HCC, of which 26 had HBV, 14 had HCV, 9 hadcryptogenic and 6 had-NASH-related cirrhosis. The annual rate of development of HCC was $1.5 \%, 3.6 \%, 0.6 \%$ and 0.46 in $\mathrm{HBV}, \mathrm{HCV}$, cryptogenic and NASH-related cirrhosis, respectively. Conclusions: The incidence of $\mathrm{HCC}$ was highest in $\mathrm{HCV}$ and lowest in NASH-related cirrhosis. These figures suggest an intermediate risk of development of HCC when compared to western countries and Japan.

KEYWORDS: hepatocellular carcinoma, non-alcoholic steatohepatitis, alpha-fetoprotein, cryptogenic cirrhosis

\section{Introduction}

The prevalence of non-alcoholic fatty liver disease (NAFLD) is increasing worldwide. ${ }^{1}$ Metabolic syndrome (MS) and diabetes mellitus (DM) are well-known risk factors of NAFLD, ${ }^{2}$ as well as of hepatic and extrahepatic malignancy. ${ }^{3}$ NALFD is broadly classified into simple steatosis and non-alcoholic steatohepatitis (NASH). ${ }^{4}$ The natural history of simple steatosis suggests that it is commonly associated with increased mortality due to cardiometabolic diseases and cancers, while NASH is associated with increased risk of development of cirrhosis and hepatocellular carcinoma (HCC). ${ }^{1,3,4}$ Overall, the natural history suggests that $5 \%$ of patients with simple steatosis can develop steatohepatitis over a period of 8 years; $25 \%$ of patients with NASH can progress to advanced fibrosis and cirrhosis. ${ }^{5-7}$ Development of HCC in patients with NAFLD 
has been well documented. ${ }^{8-10}$ A recently conducted systemic review confirms the epidemiological evidence to support an association between NAFLD/NASH and increased risk of HCC. ${ }^{11}$ Nagaoki et al. ${ }^{12}$ have shown that $15 \%$ of patients with $\mathrm{HCC}$ were not related to hepatitis B virus (HBV) or hepatitis C virus (HCV) infection. DM is one of the major risk factors in this population. This study concluded that HCC unrelated to viral infection is rising and NASH and MS are important risk factors for the same. There is an epidemic of DM and MS in Asian countries. ${ }^{13}$ Insulin resistance, which is associated with NAFLD and NASH, appears to play a significant role in hepatocarcinogenesis. ${ }^{9}$ Complications of NASH, cirrhosis and $\mathrm{HCC}$ are likely to increase in the near future.

Cirrhosis of any aetiology has been a well-known risk factor for the development of HCC. ${ }^{14-18}$ Surveillance for HCC in cirrhotic population is recommended in patients with cirrhosis of any aetiology by the American, European and Asia-Pacific Associations. ${ }^{19-21}$ The rates of development of HCC are different in different parts of the world and are dependent on the aetiology. ${ }^{22}$ On an average, HCC develops in $1 \%-6 \%$ of patients with cirrhosis of any aetiology in studies from Europe and Japan. ${ }^{23-24}$ The annual rate of development of HCC in HBVrelated cirrhosis varies from $2.5 \%$ to $6 \%$ in various studies from Taiwan, Asia and Europe. ${ }^{16,25,26}$ The annual rate of development of HCC in HCV-related cirrhosis varies from $4 \%$ to $8 \%$ in various studies from India, USA, Europe and Japan. ${ }^{25-}$ ${ }^{27}$ There is paucity of data on the annual rate of development of HCC in patients with NASH-related cirrhosis. The costeffectiveness of a surveillance programme is dependent on the threshold incidence of efficacy of surveillance as the threshold annual incidence is not known in patients with NASH-related cirrhosis. ${ }^{28}$ We planned a prospective study for determining the annual incidence of development of HCC in patients with NASH-related cirrhosis and compared it with HBV and HCVrelated cirrhosis.

\section{Methods}

A prospective observational study was conducted at a tertiary referral centre from January 2010 to October 2011. During this period, we enrolled 585 patients with liver cirrhosis. The diagnosis of cirrhosis was based on clinical, biochemical, endoscopic and histological findings whenever deemed necessary. Clinical work-up included detailed history, complete blood count, viral markers including $\mathrm{HBsAg}$, anti-HBc total, anti-HBs and anti-HCV. Work-up for autoimmune hepatitis and
Wilson's' disease (WD) was done when deemed necessary. Qualitative testing was done for HBV-DNA and HCV-RNA in patients with cryptogenic cirrhosis, and alcoholic cirrhosis was considered with standard criteria. ${ }^{-29,30}$ All patients underwent ultrasonographic examination, upper gastrointestinal (GI) endoscopy and alpha-foetoprotein (AFP) testing. We excluded all the patients with alcoholic cirrhosis and cirrhosis of rare aetiologies such as Wilson's disease, autoimmune hepatitis $(\mathrm{AIH})$, primary biliary cirrhosis (PBC) and Budd-Chiari syndrome (BCS). Patients with Child class $\mathrm{C}$ cirrhosis and those with increased risk of mortality due to other comorbid conditions such as severe coronary artery disease and renal failure were also excluded. Patients diagnosed with $\mathrm{HCC}$ at the initial presentation and those who developed HCC during the first year of surveillance were also excluded. All patients underwent regular surveillance for HCC with ultrasound and AFP testing. Abdominal ultrasound was performed by Siemens ultrasound machine using $3.5 \mathrm{MHz}$ curvilinear transducer, through the subcostal and intercostal approach. The AFP level was estimated by Axsym system (Abbot Laboratory, USA) based on the microparticle enzyme immunoassay technology. When HCC was suspected on ultrasound or on the basis of elevated AFP levels, patients were subjected to either triplephase CT scan, CT scan or magnetic resonance imaging (MRI) of the liver. Diagnosis of HCC was based on the liver tumours showing typical features of $\mathrm{HCC}$, i.e. enhancement during the arterial phase and wash-out during the portal venous phase on two imaging modalities or single imaging modality with serum AFP levels >400 ng/dL. Histology or cytology was performed only when radiological features were atypical and AFP levels were normal. ${ }^{19}$

All patients were followed up 6-monthly with ultrasound and AFP assessment. The study population included patients diagnosed to have liver cirrhosis for the first time. Previously diagnosed patients with cirrhosis were those who were diagnosed to have cirrhosis before presentation to our unit. Previously diagnosed patients with cirrhosis who were on a regular surveillance programme and attended during the study period were also included in the study. Diagnosis of HBV and HCV-related cirrhosis was made on the basis of serological markers. Diagnosis of NASH-related cirrhosis was made on the basis of exclusion of other causes of cirrhosis and either histological evidence of NASH or presence of two of the three risk factors for NASH (DM, obesity, dyslipidaemia). Diagnosis of cryptogenic cirrhosis was made when no other causes of liver disease were found and patients did not qualify for the 
diagnosis of NASH-related cirrhosis. All the subjects gave written informed consent and the study was approved by the institutional review board.

\section{Results}

A total of 585 patients with cirrhosis who fulfilled the inclusion and exclusion criteria were prospectively analysed. The mean age of these patients was $50.1 \pm 6.1$ years with men to women ratio of 4.4:1. The aetiological spectrum of this cohort was HBV 19\%, HCV 14.2\%, NASH-related 7\% and cryptogenic $17.8 \%$ cirrhosis.

More than $48 \%$ patients were already diagnosed with cirrhosis and were on a regular 6-monthly follow-up by ultrasound and AFP assessment in our unit. The remaining patients were cases of freshly diagnosed cirrhosis. All patients with Child class A and B cirrhosis without any comorbid illness were also put on a regular surveillance protocol.

The mean age of patients with HBV infection $(\mathrm{n}=111)$ was $51.8 \pm 5.6$ years; of whom $87 \%$ were negative and $90 \%$ of them were viraemic. In $\mathrm{HCV}$ infection ( $\mathrm{n}=83$ ), the mean age of patients was $57.1 \pm 7.2$ years and $76 \%$ were viraemic. The mean age of patients with cryptogenic cirrhosis ( $\mathrm{n}=104)$ was $42.5 \pm 5.7$ years while in NASH-related cirrhosis $(n=41)$, the mean age was $62.2 \pm 4.6$ years. AFP levels in all these patients at the beginning of follow-up were $<100 \mathrm{ng} / \mathrm{mL}$. Over a follow-up period 54 patients developed HCC, 26 HBV-related, 14 HCV-related, 9 cryptogenic and $6 \mathrm{NASH}$-related cirrhosis. The cumulative follow-up was $5.9 \pm 0.5$ years, $6.1 \pm 0.8$ years, $5.7 \pm 0.5,6.8 \pm 1.2$ years in $\mathrm{HBV}, \mathrm{HCV}$, cryptogenic and NASH-related cirrhosis, respectively.

About $80 \%$ of patients had early-stage HCC while $20 \%$ had advanced HCC; $67 \%$ of patients were eligible for treatment or liver transplantation. One-year survival rate in these patients was $70 \%$ and survival beyond 3 years could be seen in $20 \%$ of patients.
The incidence of HCC in various aetiological groups is shown in Table 1. The annual rate of development of HCC was $1.5 \%$ per year for $\mathrm{HBV}$-related $\mathrm{HCC}, 3.6 \%$ per year for HCVrelated HCC, $0.6 \%$ per year for cryptogenic cirrhosis and $0.46 \%$ per year for NASH-related cirrhosis.

The prevalence of DM was $21 \%$ in HBV, $20 \%$ in $\mathrm{HCV}$ and $38 \%$ in NASH-related and cryptogenic cirrhosis. About 50\% of patients with DM and HCC had a multicentric disease and/ or tumour $>5 \mathrm{~cm}$. The annual rate of development of NASHrelated cirrhosis was $0.46 \%$ per year. DM was the important risk factor in the development of $\mathrm{HCC}$ and advanced $\mathrm{HCC}$ in this population.

\section{Discussion}

The natural history of NAFLD has become evident in the recent past. Simple steatosis can progress to steatohepatitis in 5\% of patients over a period of 8 years while those who have steatohepatitis can progress to severe fibrosis and cirrhosis in $3 \%-15 \%$ of patients over a period of 5-8 years. ${ }^{5-7}$ Patients with liver cirrhosis due to NASH are at an increased risk of development of HCC; ${ }^{11}$ and $\mathrm{HCC}$ can develop in patients with NASH without cirrhosis. ${ }^{31}$ Increasing incidence of NASHrelated HCC has recently been reported from Japan. ${ }^{12} \mathrm{DM}$ and obesity are well-known risk factors for the development of HCC which are commonly seen in patients with NASH.

Our study confirms the risk of development of HCC in patients with NASH. The risk of HCC in patients with NASHrelated cirrhosis and cryptogenic cirrhosis was shown to be $0.46 \%$ and $0.6 \%$ per year, respectively which is less as compared to HCV and HBV-related HCC. Six clinic-based studies have reported the development of HCC in NASH-related or cryptogenic cirrhosis varying from $2.4 \%$ to $38 \%$ in the mean follow-up period of 3.2-10 years. ${ }^{10,32-36}$ A majority of studies have compared NASH-related or cryptogenic cirrhosis with HCC-related cirrhosis. The incidence and mortality of HCC is

Table 1: Risk of development of HCC

\begin{tabular}{llll}
\hline Aetiology & $\begin{array}{l}\text { Age, mean } \\
\text { (years) }\end{array}$ & $\begin{array}{l}\text { Follow-up period, } \\
\text { mean (years) }\end{array}$ & $\begin{array}{l}\text { Number of patients } \\
\text { developing HCC (\%) }\end{array}$ \\
\hline Hepatitis B infection $(\mathrm{n}=111)$ & $\begin{array}{l}\text { Annual rate of } \\
\text { development of } \\
\text { HCC }(\% \text { per year) }\end{array}$ \\
Hepatitis C infection $(\mathrm{n}=83)$ & $51.8 \pm 5.6$ & $5.9 \pm 0.5$ & $26(23)$ \\
Cryptogenic $(\mathrm{n}=104)$ & $57.1 \pm 7.2$ & $6.1 \pm 0.8$ & $14(17)$ \\
NASH-related $(\mathrm{n}=41)$ & $42.5 \pm 5.7$ & $5.7 \pm 0.5$ & $9(9)$ \\
\hline
\end{tabular}

$\mathrm{HCC}=$ hepatocellular carcinoma; $\mathrm{NASH}=$ non-alcoholic steatohepatitis 
much higher in HCV-related cirrhosis. ${ }^{33-34}$ Predictors of HCC in NASH-related or cryptogenic cirrhosis are body mass index (BMI), aspartate aminotransferase level, platelet count and DM. ${ }^{10}$ In our study, $38 \%$ of patients with cryptogenic cirrhosis had DM and $100 \%$ of patients had BMI $>23 \mathrm{~kg} / \mathrm{m}^{2}$.

The cost-effectiveness of a surveillance programme depends on the risk of development of HCC. Threshold incidence of the efficacy of surveillance is $>0.25$ life years gain $\% / y e a r .{ }^{28}$ Surveillance benefit is certain in chronic HBV carriers above the age of 50 years and in HBV- and HCV-related cirrhosis. Surveillance benefit has been shown in primary biliary cirrhosis and genetic haemochromatosis. ${ }^{28}$ Our data suggest surveillance benefit in patients with NASH-related or cryptogenic cirrhosis. The incidence of $\mathrm{HCC}$ in non-cirrhotic NAFLD though higher than that in the general population is not adequate enough to recommend surveillance. There has been a rapid increase in the prevalence of obesity and DM worldwide. A sharp rise in patients with NASH and its antecedent complication such as cirrhosis and $\mathrm{HCC}$ is expected in the near future.

\section{Conclusions}

This prospective study shows an increased risk of development of HCC in NASH-related cirrhosis. The risk of development of $\mathrm{HCC}$ in viral-related cirrhosis was higher when compared to NASH-related cirrhosis. The risk of HCC in cryptogenic cirrhosis is similar to that in NASH-related cirrhosis.

\section{References}

1. Amarapurkar DN, Hashimoto E, Lesmana LA, Sollano JD, Chen PJ, Goh KL, Asia-Pacific Working Party on NAFLD. How common is non-alcoholic fatty liver disease in the Asia-Pacific region and are there local differences? J Gastroenterol Hepatol. 2007;22:788-93.

2. Fan JG, Saibara T, Chitturi S, Kim B, Sung JJ, Chutaputti A, Asia-Pacific Working Party on NAFLD. What are the risk factors and settings for non-alcoholic fatty liver disease in Asia-Pacific? J Gastroenterol Hepatol. 2007;22:794-800.

3. Vuppalanchi R, Chalasani N. Nonalcoholic fatty liver disease and nonalcoholic steatohepatitis: selected practical issues in their evaluation and management. Hepatology. 2009;49:306-17.

4. Ratziu V, Bellentani S, Cortez-Pinto H, Day C, Marchesini G. A position statement on NAFLD/NASH based on the EASL 2009 special conference. J Hepatol. 2010;53:372-84.

5. Bugianesi E, Leone N, Vanni E, Marchesini G, Brunello F, Carucci $\mathrm{P}$, et al. Expanding the natural history of nonalcoholic steatohepatitis: from cryptogenic cirrhosis to hepatocellular carcinoma. Gastroenterology. 2002;123:134-40.

6. Day CP. Non-alcoholic fatty liver disease: current concepts and management strategies. Clin Med. 2006;6:19-25.

7. Bhala N, Jouness RI, Bugianesi E. Epidemiology and natural history of patients with NAFLD. Curr Pharm Des. 2013;19:5169-76.

8. Bugianesi E. Non-alcoholic steatohepatitis and cancer. Clin Liver Dis. 2007;11:191-207.

9. Starley BQ, Calcagno CJ, Harrison SA. Nonalcoholic fatty liver disease and hepatocellular carcinoma: a weighty connection. Hepatology. 2010;51:1820-32.

10. Ascha MS, Hanouneh IA, Lopez R, Tamimi TA, Feldstein AF, Zein NN. The incidence and risk factors of hepatocellular carcinoma in patients with nonalcoholic steatohepatitis. Hepatology. 2010;51:1972-8.

11. White DL, Kanwal F, El-Serag HB. Association between nonalcoholic fatty liver disease and risk for hepatocellular cancer, based on systematic review. Clin Gastroenterol Hepatol. 2012;10:1342-59.

12. Nagaoki Y, Hyogo H, Aikata H, Tanaka M, Naeshiro N, Nakahara $\mathrm{T}$, et al. Recent trend of clinical features in patients with hepatocellular carcinoma. Hepatol Res. 2012;42:368-75.

13. Yoon KH, Lee JH, Kim JW, Cho YH, Ko SH, Zimmet P, et al. Epidemic obesity and type 2 in Asia. Lancet. 2006;368:1681-8.

14. Llovet JM, Bruix J. Novel advancements in the management of hepatocellular carcinoma in 2008. J Hepatol. 2008;48:S20-S37.

15. Tang ZY, Yu YQ, Zhou XD, Yang BH, Ma ZC, Lin ZY. Subclinical hepatocellular carcinoma: an analysis of 391 patients. J Surg Oncol Suppl. 1993;3:55-8.

16. Colombo M, de Franchis R, Del Ninno E, Sangiovanni A, De Fazio C, Tommasini M, et al. Hepatocellular carcinoma in Italian patients with cirrhosis. $N$ Engl J Med. 1991;325:675-80.

17. El-Serag HB, Mason AC, Key C. Trends in survival of patients with hepatocellular carcinoma between 1977 and 1996 in the United States. Hepatology. 2001;33:62-5.

18. Luna G, Florence L, Johansen K. Hepatocellular carcinoma: a 5year institutional experience. Am J Surg. 1985;149:591-4.

19. Bruix J, Sherman M; American Association for the Study of Liver Diseases. Management of hepatocellular carcinoma: an update. Hepatology. 2011;53:1020-2.

20. Asia Pacific Working Party on Prevention of Hepatocellular Carcinoma. Prevention of hepatocellular carcinoma in the AsiaPacific region: consensus statements. J Gastroenterol Hepatol. 2010;25:657-63.

21. Omata M, Lesmana LA, Tateishi R, Chen PJ, Lin SM, Yoshida $\mathrm{H}$, et al. Asian Pacific Association for the Study of the Liver consensus recommendations on hepatocellular carcinoma. Hepatol Int. 2010;4:439-74.

22. Llovet JM, Burroughs A, Bruix J. Hepatocellular carcinoma. Lancet. 2003;362:1907-17.

23. Bosch FX, Ribes J, Borras J. Epidemiology of primary liver cancer. Semin Liver Dis. 1999;19:271-85.

24. Thompson Coon J, Rogers G, Hewson P, Wright D, Anderson R, Cramp M, et al. Surveillance of cirrhosis for hepatocellular carcinoma: systemic review and economic analysis. Health Technol Assess. 2007;11:1-206. 
25. Fattovich G, Stroffolini T, Zagni I, Donato F. Hepatocellular carcinoma in cirrhosis: incidence and risk factor. Gastroenterology. 2004;127:S35-S55.

26. [deleted - same as no. 16]Tsukuma H, Hiyama T, Tanaka S, Nakao M, Yabuuchi T, Kitamura T, et al. Risk factors for hepatocellular carcinoma among patients with chronic liver disease. N Engl J Med. 1993;328:1797-801.

27. Paul SB, Sreenivas V, Gulati MS, Madan K, Gupta AK, Mukhopadhyay S, et al. Incidence of hepatocellular carcinoma among Indian patients with cirrhosis of liver: an experience from a tertiary care center in northern India. Indian J Gastroenterol. 2007;26:274-8.

28. Sherman M. Hepatocellular carcinoma: epidemiology, surveillance, and diagnosis. Semin Liver Dis. 2010;30:3-16.

29. Anthony PP, Ishak KG, Nayak NC, Poulsen HE, Scheuer PJ, Sobin LH. The morphology of cirrhosis. Recommendation on definition, nomenclature, and classification by a working group sponsored by the World Health Organization. J Clin Pathol. 1978;31:395-414.

30. Gracia-Tsao G, Friedman S, Iredale J, Pinzani M. Now there are many (stages) where before there was one: in search of a pathophysiological classification of cirrhosis. Hepatology. 2010;51:1445-9.

31. Ertle J, Dechêne A, Sowa JP, Penndorf V, Herzer K, Kaiser G, et al. Non-alcoholic fatty liver disease progresses to hepatocellular carcinoma in the absence of apparent cirrhosis. Int $J$ Cancer. 2011;128:2436-43.

32. Ratziu V, Bonyhay L, Di Martino V, Charlotte F, Cavallaro L, Sayegh-Tainturier MH, et al. Survival, liver failure, and hepatocellular carcinoma in obesity-related cryptogenic cirrhosis. Hepatology. 2002;35:1485-93.

33. Sanyal AJ, Banas C, Sargeant C, Luketic VA, Sterling RK, Stravitz RT, et al. Similarities and differences in outcomes of cirrhosis due to nonalcoholic steatohepatitis and hepatitis C. Hepatology. 2006;43:682-9.

34. Kojima H, Sakurai S, Matsumura M, Umemoto N, Uemura $\mathrm{M}$, Morimoto H, et al. Cryptogenic cirrhosis in the region where obesity is not prevalent. World $J$ Gastroenterol. 2006;12:2080-5.

35. Yatsuji S, Hashimoto E, Tobari M, Taniai M, Tokushige $\mathrm{K}$, Shiratori K. Clinical features and outcomes of cirrhosis due to non-alcoholic steatohepatitis compared with cirrhosis caused by chronic hepatitis C. J Gastroenterol Hepatol. 2009;24:248-54.

36. Bhala N, Angulo P, van der Poorten D, Lee E, Hui JM, Saracco $\mathrm{G}$, et al. The natural history of nonalcoholic fatty liver disease with advanced fibrosis or cirrhosis: an international collaborative study. Hepatology. 2011;54:1208-16. 